\title{
Bubbling in Light Atom Salts: A New Method for Direct Visualization of Electron Beam-Induced Radiation Damage
}

\author{
W. H. Massover \\ Department of Cell Biology \& Molecular Medicine, UMDNJ - New Jersey Medical School, \\ Newark, NJ 07101-1709 \\ Transmitting electrons through protein specimens causes damaging changes to native polypeptide \\ structure (see reviews in [1-3]). Modern specimen preparation, instrumentation, and data collection \\ now limit this radiation damage to yield resolution levels of $3 \AA$ for 2 -D crystals and $7 \AA$ for single \\ particles; however, it remains very possible that the small residual radiation damage prevents \\ resolving even finer details of protein structure. At present, radiation damage to proteins in the \\ transmission electron microscope (TEM) is measured as fading of individual Bragg spots or rings \\ from crystalline specimens, or as the final resolution of a 2-D or 3-D reconstruction. If radiation \\ damage could be detected more easily, then additional experimental attention can be given to \\ finding the anti-damage conditions needed to improve specimen preservation during imaging and \\ diffraction. This study describes a new method to directly detect electron beam-induced radiation \\ damage, using a layer of dried light atom salt.
}

Chemical solutions are deposited onto a glow-discharge-treated thin carbon support film overlying a 400-mesh grid, drained, and then rapidly dried. Some grids are exposed to suspended bovine liver catalase crystals and molecules before adding the chemical. All solutions include $150 \AA$ protein-Acoated colloidal gold (Ted Pella, Inc.) as a focusing aid. Dry specimen grids are examined without cryo-protection at $100 \mathrm{kV}$ in a Tecnai-12 TEM (FEI Co.); low-dose images with beam blanking use the Tecnai/G2 software system with a cooled 4096x4096 CCD camera (Ultrascan: Gatan, Inc.).

Most light atom salts that dry into a thin amorphous layer rapidly form bubbles in the local area subjected to electron beam irradiation [4]. The bubbling response differs for different compounds in speed of initial bubble formation, size of bubbles, genesis of more bubbles with additional irradiation, and ability of small bubbles to fuse into yet larger bubbles. The bubbling response to the electron beam irradiation always is greater in thicker regions of the layer of dried salt. Very infrequently, a salt clearly shows notable reluctance to bubble (e.g., magnesium acetate [5]).

Sodium phosphate buffer $(\mathrm{pH}$ 7.0) serves well as a general indicator of electron beam-induced damage (Fig. 1). It dries into a thin film giving diffuse rings with electron diffraction, indicating its amorphous state. Even the first low-dose exposure can record some voids and small bubbles, indicating a high sensitivity to electron beam irradiation (Fig. 1). Further irradiation often causes bubble growth (Fig. 1). Subsequent images with high-dose irradiation often show a stable end-state with little further bubble formation. Protein within the buffer causes formation of a greater number of bubbles, probably indicating the presence of additional nucleation sites.

Bubbling in dry light atom salts goes through a typical sequence of nucleation, growth, fusion, and end-state. The gaseous content of these bubbles is unknown. The sequence of bubble development 
and evolution can be studied by recording series of identical low-dose exposures. The progressive changes observed with increasing electron dose necessitate recognition that images including anything beyond the first low-dose exposure are recording data from modified specimens. It is hoped that this new detection method will facilitate investigation of which chemical additives give the greatest protection against the damaging effects of electron beam irradiation [6].

\section{References}

[1] R. Henderson, Q. Rev. Biophys. 28 (1995) 171-193.

[2] R.M. Glaeser, J. Struct. Biol. 128 (1999) 3-14.

[3] R.F. Egerton, Micron 35 (2004) 399-409.

[4] W.H. Massover and P. Marsh, Ultramicrosc. 69 (1997) 139-150.

[5] W.H. Massover, Microsc. Microanal. 6 suppl. 2 (2000) 478-479.

[6] This research is supported in part by private funds.

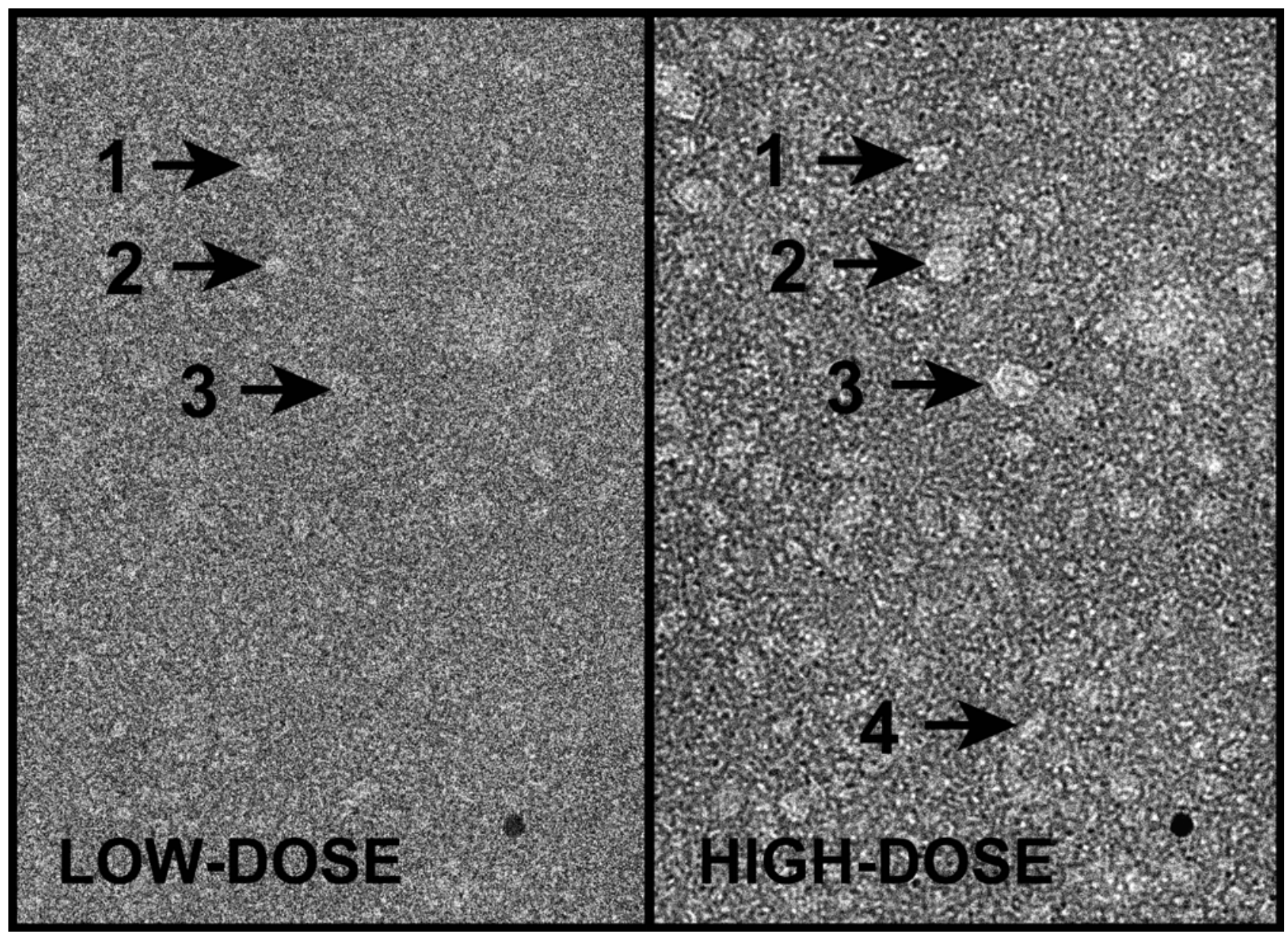

Figure 1. First low-dose and subsequent high-dose images of dried 125mM sodium phosphate buffer containing protein. Some bubbles are seen in both images, and become smaller (e.g., arrow 1). Others grow in diameter with increasing radiation dose (e.g., arrows 2 and 3). Yet other bubbles evident in the high-dose image are not detected in the preceding low-dose image (e.g., arrow 4), either because they have not yet formed or the signal/noise level is too low to permit their recognition. Dark spot at lower right of both images is a $15 \mathrm{~nm}$ colloidal gold particle. 